\title{
Helicobacter Pylori and Periodontal diseases: An update and proposal of a multidisciplinary clinical protocol
}

\author{
Sophie Marbaix ${ }^{1}$, Assem Soueidan¹, Maya Romani², Guillaume Campard ${ }^{1}$, Gilles Amador ${ }^{3}$, \\ Zahi Badran ${ }^{1}$ \\ ${ }^{1}$ Department of Periodontology (Inserm U791/UIC Odontologie/CHU de Nantes), Faculty of Dental Surgery, Nantes, France \\ ${ }^{2}$ Department of Family medicine, American University of Beirut-Medical Centre, Beirut, Lebanon \\ ${ }^{3}$ Department of Dental public health, Faculty of Dental Surgery (CHU de Nantes), Nantes, France \\ Email: zahi.badran@univ-nantes.fr
}

Received 13 June 2013; revised 14 July 2013; accepted 1 August 2013

Copyright (C) 2013 Sophie Marbaix et al. This is an open access article distributed under the Creative Commons Attribution License, which permits unrestricted use, distribution, and reproduction in any medium, provided the original work is properly cited.

\begin{abstract}
Helicobacter Pylori has been closely linked to chronic gastritis, peptic ulcers and increased risk of gastric carcinoma. Oral cavity, in particular dental plaque in periodontal pockets, may be a possible reservoir harboring $H$. Pylori, and may therefore be involved in the gastric reinfection by the bacterium, even after triple therapy regimen. This report is an update of scientific data showing the potential localization of $H$. Pylori in the oral cavity of periodontitis patients. A multidisciplinary clinical protocol combining full-mouth disinfection and triple therapy is also suggested. This protocol could permit to enhance oral $H$. Pylori eradication.
\end{abstract}

Keywords: Periodontal; Triple Therapy; Full-Mouth Disinfection

\section{INTRODUCTION}

Periodontal diseases are inflammatory chronic diseases affecting teeth supportive tissues, and have infectious bacterial etiology. It is initiated by oral bacterial biofilms developing on dental roots. Early stage of the disease is gingivitis, and is characterized by gingival inflammation (swelling, redness, bleeding $\cdots$ ). Individually susceptible patients could develop Periodontitis, which implies alveolar bone loss and periodontal pockets formation around affected teeth (Figure 1). Periodontopathogenic bacteria (PB) are mainly anaerobic, Gram negative strains developing subgingivally in periodontal pockets. This subgingival environment is particularly favorable for the survival and proliferation of non-aerobic strains. Also, many locations in the oral cavity (OC), such as the dorsum of the tongue are oxygen-poor sites. Helico-

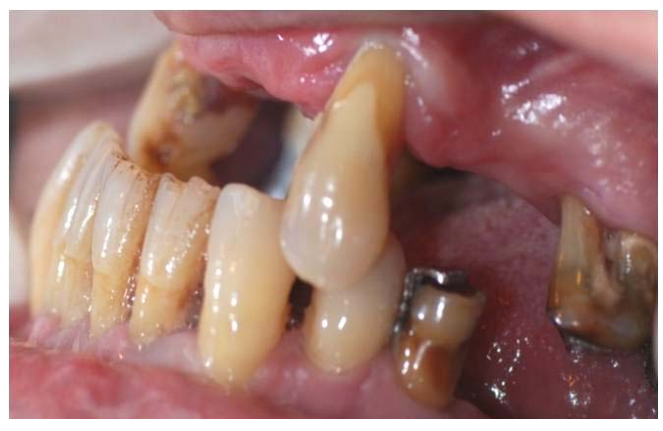

Figure 1. Periodontitis affecting teeth supportive tissues.

bacter Pylori (HP), as a gram-negative, microaerophilic, spiral-shaped bacterium; could find adequate conditions for colonization and proliferation in the OC. HP has been implicated in the etiology of gastritis and peptic ulcers. Its presence is also a risk factor for gastrointestinal cancers. Human infection by this pathogen could involve an oral route, and the $\mathrm{OC}$ is an anatomical region with a potential to harbor $H P$, in particular dental plaque (oral biofilm).

Thus, it seems conceivable that oral health status and different gingival clinical conditions may have an effect on the presence of $H P$ in oral biofilms, hence influencing the process of $H P$ gastric infection or reinfection [1,2] .

Many reports found that $H P$ could colonize the healthy OC or the subgingival periodontal pockets area. The latter could eventually serve as a bacterial reservoir. According to Savita et al. [3], subjects with periodontitis harbor a number of $H P$ significantly higher than subjects without periodontitis. Also, reinfection of the gastrointestinal tract by $H P$ from dental plaque could occur after proper eradication regimen. Triple therapy (TT) has been successfully used to eradicate gastric HP. TT is the most common first line treatment recommended for $\mathrm{H}$. Pylori 
eradication, it consists of a proton pump inhibitor (PPI) (lansoprazole $30 \mathrm{mg}$ twice daily, omeprazole $20 \mathrm{mg}$ twice daily, pantoprazole $40 \mathrm{mg}$ twice daily, rabeprazole $20 \mathrm{mg}$ twice daily) plus amoxicillin 1 gram twice daily and clarithromycin $500 \mathrm{mg}$ twice daily for 7 to 14 days. Metronidazole (500 $\mathrm{mg}$ twice daily) can be substituted for amoxicillin in penicillin-allergic patients. A longer duration of treatment ( 14 versus 7 days) may be more effective in curing infection, but this remains controversial. TT is currently considered the gold standard reaching an eradication rate up to $80 \%[1,4]$. However, in many cases, $H P$ is not completely eliminated after a single treatment, and the recurrence of the HP infection has been well-documented [5]. Therefore, the OC has been suspected to be a common source for reinfection of the stomach $[2,6]$. In fact, bacteria developing in biofilm configuration (such as in dental plaque) are much more resistant to antibiotics than freely circulating bacteria [7].

Full mouth disinfection (FMD) is a periodontal clinical protocol, combining mechanical antibacterial therapy (scaling/root planning of dental roots) and antiseptic regimen (chlorhexidine) to obtain PB eradication $[8,9]$. The mechanical debridement of all teeth is performed within $24 \mathrm{~h}$, in order to eradicate rapidly PB present in dental biofilms, and thus preventing reinfection of treated sites. Also, the adjunction of a systemic antibiotic prescription to FMD (amoxicillin/metronidazole or azithromycin) has been found to successfully enhance clinical outcomes [10-12].

This article focuses on the specific association of periodontal status and oral HP in the published scientific literature. The rationale of considering periodontitis as a risk factor for $H P$ infections/reinfection will also be discussed, with an emphasis on the eventual clinical implications of this assumption. Finally, we suggest the merging of both FMD and TT regimen, in order to enhance periodontal therapy outcome as well as eradication of $H P$.

\section{HELICOBACTER PYLORI AND PERIODONTAL DISEASES}

\subsection{Helicobacter Pylori in Periodontally Ill and Healthy Patients}

Several studies have evaluated the correlation between the prevalence of HP and periodontal diseases. Conflicting results could be observed in the literature, this could be attributed to the diversity of the studied populations, sample collection methods, and the specificity of the methods used for HP detection [2,13]. Although it may affect the presence of $H P$ in dental plaque, few studies have separately investigated supra and subgingival plaque, which corresponds to different microenvironments when availability of nutrients, oxygen and host mechanisms are considered [14].

Souto et al. [2] performed a full-mouth clinical ex- amination (six sites per tooth) to determine periodontal health status and examined subjects with no history of gastric symptoms, their results showed a higher prevalence of HP in subgingival biofilm of subjects with periodontitis compared to individuals with periodontal health (50\% versus $11 \%$ respectively), suggesting that periodontal pockets and inflammation may favor $H P$ colonization. Another study by Riggio and Lennon [15] examined the presence of $H$. Pylori in subgingival plaque (sites with periodontal pocket depth $\geq 5 \mathrm{~mm}$ ) of periodontitis patients with no symptoms of gastritis or peptic ulcer disease. This study found that $38 \%$ of subjects with deep periodontal pockets were positive for HP. Consequently, the authors concluded that subgingival plaque in individuals presenting periodontitis may function as a reservoir for $H P$.

Gebara et al. [14], found that more than $26 \%$ of $H P$ was detected in the OC of periodontitis patients (all positive for gastric $H P$ ), periodontitis and gingivitis showed a high prevalence; however, no control group with healthy patients were included. Al Asqah et al. [13] found that periodontitis patients had a significantly higher percentage of HP in subgingival plaque samples in comparison to periodontally healthy controls ( $79 \%$ versus $43 \%$ respectively). Other studies failed to detect HP in the subgingival plaque $[16,17]$ or found no association between periodontal status and the prevalence of the bacterium in OC [18].

\subsection{Relation between Gastric Infection and Periodondal Disease}

Umeda et al. [19] showed that more than $40 \%$ of patients with history of gastritis or peptic ulcers had periodontal pockets $(>4 \mathrm{~mm})$. Bruce et al. (54) found that periodontal pockets $\geq 5 \mathrm{~mm}$ are associated with increased odds of HP seropositivity. Also, a large epidemiological investigation performed over 10,000 subjects [20], found a positive link between HP associated gastric infection and periodontal diseases. The latter may facilitate the $H P$ oro-gastric transmission and colonization of the bacteria in the digestive tract. Also, periodontitis microenvironment conditions could be favorable for HP multiplication, thus increasing sufficiently to cause gastric infection $[21,22]$.

On the other hand, many studies [23-25] failed to establish a positive correlation between periodontal disease and gastric infection.

\section{TRIPLE THERAPY AND ORAL HELICOBACTER PYLORI}

\subsection{Effect of Triple Therapy on Oral $H P$}

It is still unclear whether TT affects $H P$ in dental plaque. Song et al. [26] found plaque samples negative for $H P$ 
only in patients who had recently been treated with TT. But many reports, found a persistent oral HP after TT [27-29]. More recently, a meta-analysis [30] confirmed the close relationship between HP infection in the OC and the stomach, and concluded that oral HP is more difficult to eradicate than gastric $H P$ and it may be a source of reinfection. On the other hand, Umeda et al. [19] reported that subjects with periodontal pockets retained $H P$ in the $\mathrm{OC}$ even after eradication of the bacterium from the stomach. Similarly, Gebara et al. [1] showed that $60 \%$ of subjects with periodontal disease were still positive for $H P$ DNA in their OC, whereas only $10 \%$ were positive in the stomach after triple therapy. In a same way, Zarik et al. [5] revealed that 3 months after triple therapy in the group of participants who did not harbor $H P$ in periodontal pockets, the eradication rate was $87.4 \%$. In contrast, the group positive for HP in the subgingival plaque exhibited a significantly lower rate of eradication $(47.6 \%)$. Also, polyantibiotic systemic therapy was considerably less efficient in the eradication of HP from periodontal pockets, compared with its elimination in the stomach (33\% and $87 \%$ respectively).

\subsection{Effect of Periodontal Therapy on Prevention of Gastric Helicobacter Pylori Recurrence}

Few studies investigated the effect of periodontal therapy on eradication of gastric HP. Zarik et al. [5], evaluated the effectiveness of periodontal treatment and TT in achieving complete elimination of gastric HP infection. Participants positive for HP in the subgingival biofilm, who had undergone basic periodontal therapy and a TT regimen, showed a higher elimination rates compared to the group who underwent TT alone $(77.3 \%$ and $47.6 \%$ respectively), thus suggesting that systemic antibiotic polytherapy alone is not as effective in preventing the reappearance of gastric $H P$ in subjects positive for the oral form of HP. A meta-analysis [31] concluded that periodontal therapy reduces the relative risk of persistence of gastric $H P$ by $63 \%$. Similar results were found in a study with a two years follow-up [32].

Recently, a case series of 9 patients [33], investigated whether full mouth ultrasonic debridement can eradicate persistent oral HP after TT. Periodontal debridement was able to eradicate $H P$ from $88.3 \%$ of patients with gingivitis, while it failed in eradicating the bacteria from the $\mathrm{OC}$ of periodontitis group. Within the limits of this case series, a single session of oral hygiene instruction combined with full mouth periodontal debridement seemed to promote a positive effect in eradicating $H P$ only from supragingival sites.

\section{CLINICAL MULTIDISCIPLINARY MANAGEMENT PROPOSAL}

Actual consensus in Periodontology suggests that antibi- otic regimen should be started immediately after mechanical periodontal treatment [34]. Thus, a complete elimination of calculus and disorganization of bacterial biofilms must be achieved prior to any antibiotic therapy in order to disrupt the biofilm configuration forming a barrier to antibiotic molecules. For periodontal treatment we suggest a full mouth disinfection (FMD) approach, as described in the periodontal literature [9]. The FMD consists of a total scaling/mechanical debridement of teeth root surfaces. An antiseptic irrigation of the pockets, the dorsum of the tongue and the tonsils is also performed [8]. The whole procedure should be finalized in one session (or two sessions within $24 \mathrm{~h}$ ). Immediately after FMD, an antibiotic regimen (+ antiseptic chlorhexidine mouth-washes/oral gels) has been found to significantly enhance periodontal outcome [10]. A combination of amoxicillin/metronidazole is the most tested combination after FMD [11]. On the other hand, clarithromycin has shown an excellent availability in the gingival area [35]. Hence, the antibiotic regimen in TT could substitute periodontal prescription as soon as the mechanical treatment is accomplished. This approach could enhance PP and $H P$ elimination (in the $\mathrm{OC}$ and the gastric tract). In fact, the antibiotics used to eradicate $H P$ are also efficient against the anaerobic PB. Hence, the merging of the periodontal FMD protocol and the TT (Figure 2) permits to treat both pathologies in an optimal manner, and could significantly boost oral HP eradication.

For maintaining long-term results, routine dental/periodontal supportive therapy and daily oral hygiene procedures should be performed without interruption [6].

\section{CONCLUSION}

Although contradictory results are found in the scientific

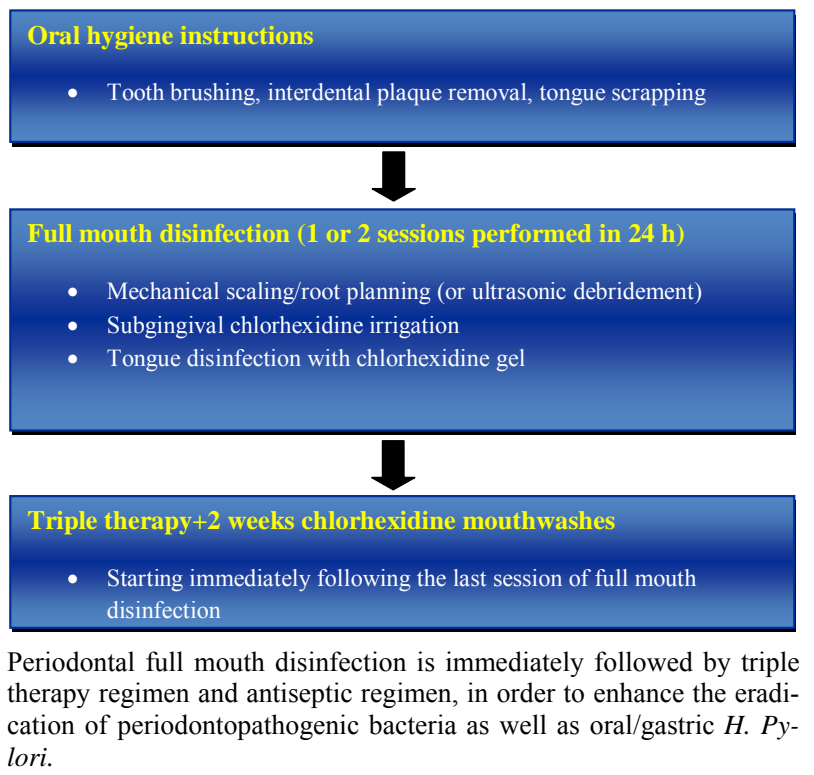

Figure 2. Multidisciplinary clinical protocol. 
literature, many reports have associated periodontal disease (periodontal pockets formation) to an increased prevalence of oral HP. Its bacterial load is increased by periodontal disease and periodontal pockets formation. The assumption that subgingival oral biofilm could act as a suitable reservoir for HP is plausible and supported by consistent evidence from preliminary clinical trials. The presence of oral HP in patients with periodontal disease (Periodontitis and gingivitis) may cause refractoriness of gastric infection to triple therapy alone [5,31].

The mechanisms that confer the ability of HP to escape systemic treatment are not well understood, but there is evidence indicating that biofilms provide defense from the host immunological response and the action of antimicrobials agents [36]. Hence, antimicrobials cannot be efficient if the biofilm is not mechanically disrupted [37] shortly prior to prescription. The subgingival dental biofilm is particularly suspected, due to the favorable micro-ecological conditions provided by the periodontal pockets. The biofilm is known to provide protection from the host's immunological response, and bacteria growing in biofilms exhibit high resistance to antimicrobial agents [5]. We therefore suggest a simple multidisciplinary clinical management protocol, merging the triple therapy to periodontal mechanical treatment and chemical antiseptic disinfection. A joint coordination between the periodontist and the gastroenterologist is a perquisite for the success of this treatment regimen. Adopting this protocol has two objectives; treating periodontal disease and enhancing the efficacy of the anti HP therapy.

On the other hand, it should be considered that the efficacy of non-surgical treatment modalities is related to the pocket depth. Hence, surgical periodontal procedures like open flap debridement could be needed in some cases to eliminate deep bacterial biofilms potentially harboring HP [33]. To our knowledge, no research has been conducted in this field.

In conclusion, the efficacy of the combined proposed protocol should be investigated in future controlled, randomized clinical trials. If confirmed, it could be integrated in the standard clinical management of HP infections.

\section{REFERENCES}

[1] Gebara, E.C., Faria, C.M., Pannuti, C., Chehter, L., Mayer, M.P. and Lima, L.A. (2006) Persistence of Helicobacter pylori in the oral cavity after systemic eradication therapy. Journal of Clinical Periodontology, 33, 329-333. doi:10.1111/j.1600-051X.2006.00915.X

[2] Souto, R. and Colombo, A.P. (2008) Detection of Helicobacter pylori by polymerase chain reaction in the subgingival biofilm and saliva of non-dyspeptic periodontal patients. Journal of Periodontology, 79, 97-103. doi:10.1902/jop.2008.070241

[3] Sambashivaiah, S., Bilichodmath, S., Nanjaiah, N. and Kulal, R. (2011) Helicobacter pylori in periodontal pockets of chronic periodontitis patients with and without type II diabetes mellitus: A randomized controlled trial.

[4] Bago, I., Plecko, V., Aurer, A., Majstorovic, K. and Budimir, A. (2011) The effectiveness of systemic eradication therapy against oral Helicobacter pylori. Journal of Oral Pathology \& Medicine, 40, 428-432. doi:10.1111/j.1600-0714.2010.00989.x

[5] Zaric, S., Bojic, B., Jankovic, L., Dapcevic, B., Popovic B., Cakic, S. and Milasin, J. (2009) Periodontal therapy improves gastric Helicobacter pylori eradication. Journal of Dental Research, 88, 946-950. doi:10.1177/0022034509344559

[6] Jia, C.L., Jiang, G.S., Li, C.H. and Li, C.R. (2009) Effect of dental plaque control on infection of Helicobacter pylori in gastric mucosa. Journal of Periodontology, $\mathbf{8 0}$, 1606-1609. doi:10.1902/jop.2009.090029

[7] Dye, B.A., Kruszon-Moran, D. and McQuillan, G. (2002) The relationship between periodontal disease attributes and Helicobacter pylori infection among adults in the United States. The American Journal of Public Health, 92, 1809-1815. doi:10.2105/AJPH.92.11.1809

[8] Quirynen, M., Mongardini, C., de Soete, M., Pauwels, M., Coucke, W., van Eldere, J. and van Steenberghe, D. (2000) The role of chlorhexidine in the one-stage full-mouth disinfection treatment of patients with advanced adult periodontitis. Long-term clinical and microbiological observations. Journal of Clinical Periodontology, 27, 578589. doi:10.1034/j.1600-051x.2000.027008578.x

[9] Quirynen, M., Mongardini, C., Pauwels, M., Bollen, C.M., Van Eldere, J. and van Steenberghe, D.(1999) One stage full-versus partial-mouth disinfection in the treatment of chronic adult or generalized early-onset periodontitis. II. Long-term impact on microbial load. Journal of Periodontology, 70, 646-656. doi:10.1902/jop.1999.70.6.646

[10] Aimetti, M., Romano, F., Guzzi, N. and Carnevale, G. (2012) Full-mouth disinfection and systemic antimicrobial therapy in generalized aggressive periodontitis: A randomized, placebo-controlled trial. Journal of Clinical Periodontology, 39, 284-294. doi:10.1111/j.1600-051X.2011.01795.x

[11] Cionca, N., Giannopoulou, C., Ugolotti, G. and Mombelli, A. (2009) Amoxicillin and metronidazole as an adjunct to full-mouth scaling and root planing of chronic periodontitis. Journal of Periodontology, 80, 364-371. doi:10.1902/jop.2009.080540

[12] Gomi, K., Yashima, A., Nagano, T., Kanazashi, M., Maeda, N. and Arai, T. (2007) Effects of full-mouth scaling and root planing in conjunction with systemically administered azithromycin. Journal of Periodontology, 78, 422429. doi:10.1902/jop.2007.060247

[13] Al Asqah, M., Al Hamoudi, N., Anil, S., Al Jebreen, A. and Al-Hamoudi, W.K. (2009) Is the presence of Helicobacter pylori in dental plaque of patients with chronic periodontitis a risk factor for gastric infection? Canadian Journal of Gastroenterology, 23, 177-179.

[14] Gebara, E.C., Pannuti, C., Faria, C.M., Chehter, L., Mayer, M.P. and Lima, L.A. (2004) Prevalence of Helico- 
bacter pylori detected by polymerase chain reaction in the oral cavity of periodontitis patients. Oral Microbiology and Immunology, 19, 277-280. doi:10.1111/j.1399-302X.2004.00153.x

[15] Riggio, M.P. and Lennon, A. (1999) Identification by PCR of Helicobacter pylori in subgingival plaque of adult periodontitis patients. Journal of Medical Microbiology, 48, 317-322. doi:10.1099/00222615-48-3-317

[16] Asikainen, S., Chen, C. and Slots, J. (1994) Absence of Helicobacter pylori in subgingival samples determined by polymerase chain reaction. Oral Microbiology and Immunology, 9, 318-320. doi:10.1111/j.1399-302X.1994.tb00079.x

[17] Silva, D.G., Stevens, R.H., Macedo, J.M., Albano, R.M., Falabella, M.E., Fischer, R.G., Veerman, E.C. and Tinoco, E.M. (2010) Presence of Helicobacter pylori in supragingival dental plaque of individuals with periodontal disease and upper gastric diseases. Archives of Oral Biology, 55, 896-901. doi:10.1016/j.archoralbio.2010.06.018

[18] Burgers, R., Schneider-Brachert, W., Reischl, U., Behr, A., Hiller, K.A., Lehn, N., Schmalz, G. and Ruhl, S. (2008) Helicobacter pylori in human oral cavity and stomach. European Journal of Oral Sciences, 116, 297-304. doi:10.1111/j.1600-0722.2008.00543.x

[19] Umeda, M., Kobayashi, H., Takeuchi, Y., Hayashi, J., Morotome-Hayashi, Y., Yano, K., Aoki, A., Ohkusa, T. and Ishikawa, I. (2003) High prevalence of Helicobacter pylori detected by PCR in the oral cavities of periodontitis patients. Journal of Periodontology, 74, 129-134. doi:10.1902/jop.2003.74.1.129

[20] Bielanski, W. (1999) Epidemiological study on Helicobacter pylori infection and extra gastroduodenal disorders in Polish population. Journal of Physiology and Pharmacology, 50, 723-733.

[21] Czesnikiewicz-Guzik, M., Bielanski, W., Guzik, T.J., Loster, B. and Konturek, S.J. (2005) Helicobacter pylori in the oral cavity and its implications for gastric infection, periodontal health, immunology and dyspepsia. Journal of Physiology and Pharmacology, 56, 77-89.

[22] Czesnikiewicz-Guzik, M., Karczewska, E., Bielanski, W., Guzik, T.J., Kapera, P., Targosz, A., Konturek, S.J. and Loster, B. (2004) Association of the presence of Helicobacter pylori in the oral cavity and in the stomach. Journal of Physiology and Pharmacology, 55, 105-115.

[23] Anand, P.S., Nandakumar, K. and Shenoy, K.T. (2006) Are dental plaque, poor oral hygiene, and periodontal disease associated with Helicobacter pylori infection? Journal of Periodontology, 77, 692-698. doi:10.1902/jop.2006.050163

[24] Berroteran, A., Perrone, M., Correnti, M., Cavazza, M.E., Tombazzi, C., Goncalvez, R. and Lecuna, V. (2002) Detection of Helicobacter pylori DNA in the oral cavity and gastroduodenal system of a Venezuelan population. Journal of Medical Microbiology, 51, 764-770.

[25] Hardo, P.G., Tugnait, A., Hassan, F., Lynch, D.A., West, A.P., Mapstone, N.P., Quirke, P., Chalmers, D.M., Kowolik, M.J. and Axon, A.T. (1995) Helicobacter pylori infection and dental care. Gut, 37, 44-46.

\section{doi:10.1136/gut.37.1.44}

[26] Song, Q., Lange, T., Spahr, A., Adler, G. and Bode, G. (2000) Characteristic distribution pattern of Helicobacter pylori in dental plaque and saliva detected with nested PCR. Journal of Medical Microbiology, 49, 349-353.

[27] Czesnikiewicz-Guzik, M., Loster, B., Bielanski, W., Guzik, T.J., Konturek, P.C., Zapala, J. and Konturek, S.J. (2007) Implications of oral Helicobacter pylori for the outcome of its gastric eradication therapy. Journal of Clinical Gastroenterology, 41, 145-151. doi:10.1097/01.mcg.0000225654.85060.3d

[28] Gurbuz, A.K., Ozel, A.M., Yazgan, Y., Celik, M. and Yildirim, S. (2003) Oral colonization of Helicobacter pylori: Risk factors and response to eradication therapy. South African Medical Journal, 96, 244-247. doi:10.1097/01.SMJ.0000051069.50950.2B

[29] Pytko-Polonczyk, J., Konturek, S.J., Karczewska, E., Bielanski, W. and Kaczmarczyk-Stachowska, A. (1996) Oral cavity as permanent reservoir of Helicobacter pylori and potential source of reinfection. Journal of Physiology and Pharmacology, 47, 121-129.

[30] Zou, Q.H. and Li, R.Q. (2011) Helicobacter pylori in the oral cavity and gastric mucosa: a meta-analysis. Journal of Oral Pathology \& Medicine, 40, 317-324. doi:10.1111/j.1600-0714.2011.01006.x

[31] Bouziane, A., Ahid, S., Abouqal, R. and Ennibi, O. (2012) Effect of periodontal therapy on prevention of gastric Helicobacter pylori recurrence: A systematic review and meta-analysis. Journal of Clinical Periodontology, 39, 1166-1173. doi: $10.1111 /$ jepe. 12015

[32] Tarullo, A., Tattoli, M. and Cagiano, R. (2001) Persistent eradication of Helicobacter pylori after systemic politherapy associated with periodontal pockets treatment with metronidazole and calcium sulphate. European Review for Medical and Pharmacological Sciences, 5, 127129.

[33] Escobar, E.C., Chambrone, L., Pannut, C.M., Mayer, M.P.A. and Lima, 1.A. (2013) Full mouth ultrasonic debridement in Helicobacter Pylori eradication from the oral cavity: A case series. Canadian Journal of Dental Hygiene, 47, 39.

[34] Sanz, M. and Teughels, W. (2008) Innovations in nonsurgical periodontal therapy: Consensus Report of the Sixth European Workshop on Periodontology. Journal of Clinical Periodontology, 35, 3-7. doi:10.1111/j.1600-051X.2008.01256.x

[35] Burrell, R.C. and Walters, J.D. (2008) Distribution of systemic clarithromycin to gingiva. Journal of Periodontology, 79, 1712-1718. doi:10.1902/jop.2008.080013

[36] Socransky, S.S. and Haffajee, A.D. (2002) Dental biofilms: Difficult therapeutic targets. Periodontology, 28, 12-55. doi:10.1034/j.1600-0757.2002.280102.x

[37] Herrera, D., Alonso, B., Leon, R., Roldan, S. and Sanz, M. (2008) Antimicrobial therapy in periodontitis: The use of systemic antimicrobials against the subgingival biofilm. Journal of Clinical Periodontology, 35, 45-66. doi:10.1111/j.1600-051X.2008.01260.X 